\title{
Determination of the sticking coefficient of energetic hydrocarbon molecules by molecular dynamics
}

\author{
Klaus Tichmann, Udo von Toussaint*, Wolfgang Jacob \\ Max-Planck-Institut für Plasmaphysik, EURATOM Association \\ Boltzmannstr. 2, 85748 Garching, Germany
}

\begin{abstract}
The sticking coefficient of hydrocarbon species is a key quantity that influences the growth process of amorphous hydrocarbon layers. To extend the very limited database for low impact energies, classical molecular dynamics simulations were performed, determining the sticking coefficients of $\mathrm{CH}_{x}(x=0 \ldots 4)$ with kinetic energies between 5 and $100 \mathrm{eV}$. Similar simulations are performed with hydrogen substituted by deuterium. Additionally, analytical formulas are presented that fit the data very well and can be used to interpolate the simulation results.
\end{abstract}

\section{INTRODUCTION}

The divertors of future fusion experiments will have to withstand extreme conditions. The current planning for ITER uses carbon tiles in the divertor [1], that should withstand the power load but will get eroded by hydrogen from the plasma. In this process, hydrocarbon molecules are created which can cause a subsequent formation of redeposited layers [2]. In later stages of the ITER experimental program, these hydrocarbons will contain tritium and tritium retention limits the usable operation time of a fusion experiment. Therefore, it is of vital importance to know where these layers form, how fast they are deposited and how the layers can be removed.

Transport simulations codes such as ERO [3] or DIVIMP3D [4] address these questions, based on assumptions about sputtering of the surface, transport of impurities in the boundary plasma and sticking of particles to the surrounding surfaces. The sticking of hydrocarbon fragments depends on the identity of the fragment, its kinetic energy and its angle of incidence. In the end, this quantity determines how many surface collisions the particle will survive before being deposited. Using classical molecular dynamics (MD) we simulate the impact of different hydrocarbon species on a suitable hydrocarbon surface. By following the trajectories of individual atoms, the sticking coefficient, the amount of sputtered sample material and the species emitted due to the projectile impact are determined. To take into account the influence of the sample surface, the simulations are done on an amorphous hydrocarbon sample that is similar to the kind of material that grows from the impacting projectiles.

Similar studies were performed by Alman et al. [5] and Ohya et al. [6]. Alman et al. [5] used an amorphised graphite sample, focussing on low projectile energies $(25 \mathrm{meV}-50 \mathrm{eV})$ with sequential bombardment, using the Brenner hydrocarbon potential [7]. The simulations by Ohya et al. [6] operated on a sample of hydrogenated and amorphised graphite using the Juslin potential [8] and projectile energies up to $100 \mathrm{eV}$. The database built by these publications was extended with respect to angle of incidence as well as to higher projectile energies. In addition, a possible isotope effect for deuterated species was investigated.

\section{THE STUDIED QUANTITIES}

Unlike the case of atoms, there is no generally accepted definition for the sticking coefficient of molecules. To define a sticking coefficient, one must take note of what can happen when the projectile hits the surface. The two limiting cases are that the projectile is reflected and that it sticks completely. For molecular projectiles there are additional interaction channels where the projectile breaks up and some constituents (or nothing) stay on the surface. In addition to that, atoms can be removed from the surface by physical or chemical sputtering. Due to this large number of processes, a single sticking coefficient is insufficient. The hydrogen content of the deposited layer is to a large extent determined by the energy of the projectiles, while the growth of the layer is limited by the sticking of carbon. Therefore, only the sticking coefficient of carbon is studied here, since the sticking of hydrogen can be derived from that, using additional knowledge about the composition of the redeposited layers [9]. The following three quantities are defined:

Gross sticking coefficient $S_{\text {gross }}$ : The number of projectile carbon atoms deposited per incident carbon atom

Sputter yield $Y$ : The number of sputtered carbon atoms per incident carbon atom

Net sticking coefficient $S_{\text {net }}$ : The net change of the number of carbon atoms in the sample per incident carbon atom. This quantity is the difference of the first two.

All quantities are defined relative to the number of incident carbon atoms, i.e., carbon atoms that reach the surface. This is especially important if projectiles contain more than one carbon atom, as in this case the number of carbon atoms is not the same as the number of incident projectiles.

Only the net sticking coefficient can be directly compared to experimental values. For comparison with other simulation studies, also the gross sticking coefficient and the 
sputtering yield are of relevance. For transport simulations, the particles that are emitted from the surface are important as well.

\section{SIMULATION PROCEDURE}

When deposition starts on a clean substrate, at first an intermediate layer grows with a strongly substrate dependent growth rate. As this intermediate layer grows, the influence of the original substrate diminishes and the growth rate approaches a steady state value that does not depend on the substrate any more. If an intermediate layer can form at all, the steady state growth rate will always dominate the total deposition rate in the long run. Therefore, studying the steady-state layer growth will cover the more important case for many applications (and the results will be mostly independent of the chosen substrate material).

For irradiation with energetic hydrocarbons, it is known that the material deposited will be amorphous hydrocarbon or a-C:H [10].

An MD sample was created with chemical composition, density and radial structure function that were determined experimentally from real a- $\mathrm{C}: \mathrm{H}$ films [9]. A detailed description of the MD sample production process was given in [11]. The MD sample consists of 986 atoms (592 carbon, 394 hydrogen) and has outer dimensions of $1.4 \times 1.4 \times 2.8 \mathrm{~nm}^{3}$ with periodic boundary conditions in $x$ and $y$ directions. To make sure that the MD sample is in equilibrium, it was annealed for $2 \mathrm{~ns}$ of simulated time.

If the flux of projectiles to the surface is very low, it can be assumed that the sample surface is in equilibrium before the next impact occurs. In this case, the very same sample can be used for all simulations, since it is assumed that the time between incoming projectiles is long enough that they do not affect each other. This approximation can only yield meaningful results if the sample is in thermal equilibrium and in steady-state with respect to layer growth. While thermal equilibrium can be reached by giving the sample enough simulated time, steady-state with respect to the layer growth cannot be guaranteed to hold unless the sample is grown under the very same conditions as those studied, which cannot be achieved in reasonable time. The MD sample used here is a good approximation to the steady state growth so that it can be assumed that the impacts are independent.

All simulations are started from the equilibrated sample, using the approximation of low flux stated above. Since the individual simulations are thus independent of each other, they can be done in parallel, speeding up the calculation immensely.

The projectiles were equilibrated in a separate simulation. For the main simulation, the projectiles were placed on a plane $0.4 \mathrm{~nm}$ above the topmost atom of the sample. The projectile's velocity vector was set according to the selected irradiation energy and angle of incidence. The angle of incidence was varied from perpendicular incidence to $88^{\circ}$. The projectiles were randomly oriented for every impact to average out possible angular dependencies. Dependencies like this were observed by Sharma et al. for the sticking of $\mathrm{CH}[14]$. An analysis of the functional dependence of the sticking coefficient of $\mathrm{CH}_{x}$ $(x=0 \ldots 4)$ on the angle of incidence at a fixed projectile energy was already published elsewhere [12]. These data represent a subset of the simulation results presented in this article.

The projectile energy was varied between 5 and $150 \mathrm{eV}$. The low energy limit was chosen so that the kinetic energy of the projectiles is always well above reasonable values for the thermal energy of the projectile, so that the effect of thermal excitations of vibrational [13] or rotational [14] degrees of freedom of the projectile can be neglected. By setting the internal energy of the projectiles to zero, the dimension of the parameter space can be greatly reduced. All remaining degrees of freedom were set to random numbers for each of the 1000 independent impacts.

The simulation was run for $10 \mathrm{ps}$ using a variable time step of up to $0.25 \mathrm{fs}$. The hydrocarbon interactions were calculated from the Brenner hydrocarbon potential [7] with additions from Beardmore [15] as implemented by Nordlund in the hcparcas code [16].

In order to determine the differences between protonated hydrocarbons and their deuterated counterparts, the same simulations were repeated with the mass of all hydrogen atoms set to $2 \mathrm{amu}$. No additional modifications were introduced to the potential, so the chemical behaviour of deuterium is identical to that of hydrogen.

\section{ANALYSIS}

After the simulation run is finished, the number of sputtered atoms is computed based on the network of chemical bonds in the system. Since the simulation itself does not yield any information on the chemical bonds in the system, they were determined from the distances of the individual atoms. Atoms are considered bound to each other if their distance is less than $0.2 \mathrm{~nm}$. The bonds derived from the atoms distances form networks that group those atoms that are directly or indirectly bound to each other. Groups that are at least partially located within the limits of the original sample at the end of the simulation are considered bound to the surface. All others are counted as reflected or sputtered, depending on their origin.

Having determined which atoms have left the surface, the remaining quantities can be calculated. For the list of emitted species, the same bond network is used. The number of emitted particles was counted over 1000 simulation runs with identical impact parameters. 


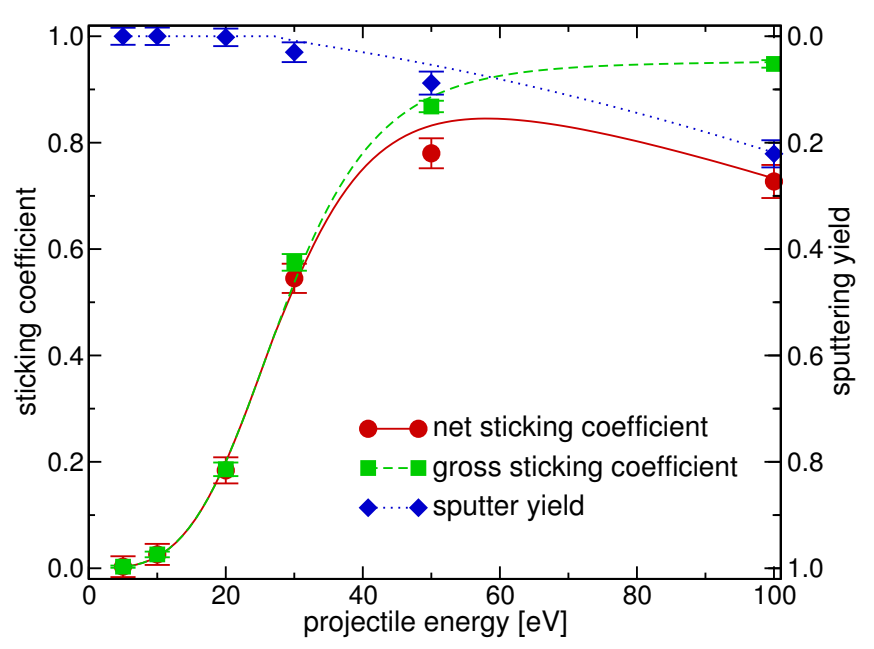

FIG. 1: Net and gross sticking (left axis) and sputtering yield (right axis, inverted scale) plotted versus the energy of the impinging $\mathrm{CH}_{4}$ molecule at normal incidence. The lines represent the interpolation functions as discussed in section VI, namely Eckstein's formula [17] for sputtering, a rational bezier spline [18] for gross sticking and the sum of both for the net sticking coefficient.

\section{RESULTS}

The experimentally observable quantity is the net sticking coefficient $S_{\text {net }}=S_{\text {gross }}-Y$, that takes sticking $\left(S_{\text {gross }}\right)$, reflection and sputtering $(Y)$ into account. As an example, the gross sticking, sputtering and the net sticking of $\mathrm{CH}_{4}$ at normal incidence are plotted versus the incident energy in figure 1 . The net sticking coefficient (circles) is determined from the gross sticking of the projectile itself (squares) and the sputtering of sample atoms (diamonds, plotted on the reversed right axis). Below the sputtering threshold of about $20 \mathrm{eV}$, no sputtered atoms were observed and the net sticking is determined only by the sticking of the projectile. The projectile sticking is very small at energies up to $20 \mathrm{eV}$, increases quickly to about 0.8 between 20 and $50 \mathrm{eV}$ and approaches 1 for energies above $50 \mathrm{eV}$. Sputtering of the sample becomes noticeable at $30 \mathrm{eV}$ and increases with energy. Since the projectile sticking saturates for energies higher than $50 \mathrm{eV}$ while the sputtering keeps increasing with energy, the net sticking coefficient has a maximum at about $50 \mathrm{eV}$.

The net sticking coefficient of different $\mathrm{CH}_{x}(x=1-4)$ projectiles is plotted in figure 2 . In the high energy domain above $50 \mathrm{eV}$, where the gross sticking is in saturation, the net sticking coefficient of all projectile species shows a very similar, decreasing behaviour due to increasing sputtering yield. In this region, the net sticking coefficient of different projectile species varies by less then $10 \%$. The net sticking coefficient of $\mathrm{CH}$ and $\mathrm{CH}_{2}$ increases almost linearly with decreasing energy over almost the full investigated energy range, reaching values

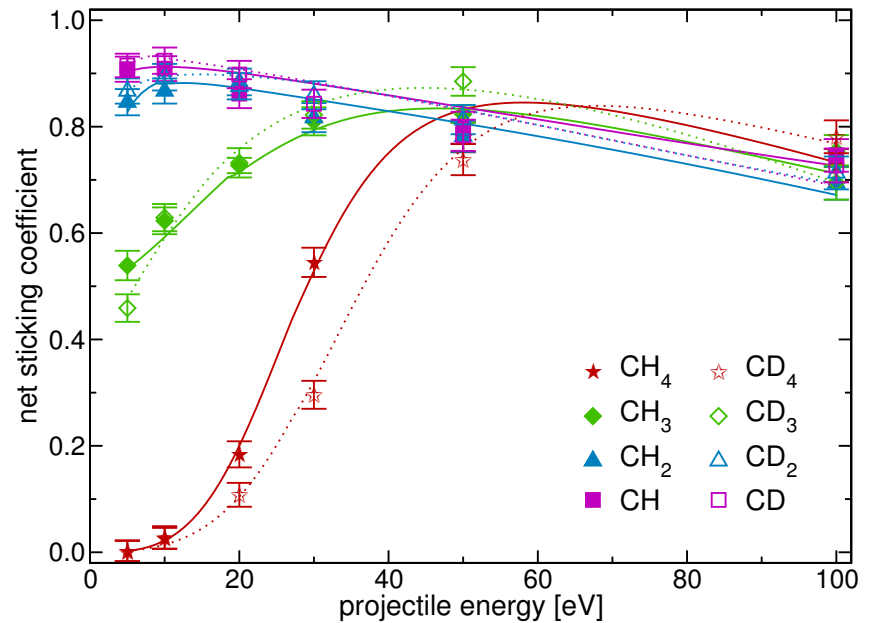

FIG. 2: Net sticking coefficients for $\mathrm{CH}_{x}$ and $\mathrm{CD}_{x}$ projectiles with $x=1 \ldots 4$, plotted against the kinetic energy of the projectile (normal incidence). The lines are fits to the data, see section VI. The lines depict the interpolation functions discussed in section VI with solid lines for protinated and dotted for deuterated projectiles.

of about 0.9 at the lowest energies. For $\mathrm{CH}_{3}$, the net sticking coefficient decreases with decreasing energy at energies below $50 \mathrm{eV}$ to about 0.5 at $5 \mathrm{eV}$, while for $\mathrm{CH}_{4}$, it decreases strongly for energies below $50 \mathrm{eV}$ and approaches 0 at less than $10 \mathrm{eV}$.

In addition to the normal $\mathrm{CH}_{x}$ species, the sticking coefficient of the corresponding deuterated species $\left(\mathrm{CD}_{x}\right)$ is plotted in figure 2 with open symbols. For most of the projectile species, the differences in sticking coefficient between the protonated and the deuterated variant are smaller than the statistical uncertainties. As an exception to that, the sticking coefficient of $\mathrm{CH}_{4}$ is significantly larger than the sticking coefficient of $\mathrm{CD}_{4}$ between $20 \mathrm{eV}$ and $50 \mathrm{eV}$. When plotting the same data versus the energy of the carbon atom (see figure 3), the differences between $\mathrm{CH}_{4}$ and $\mathrm{CD}_{4}$ vanish completely. For the $\mathrm{CH}_{x}(x=1-3)$ species, the differences between protium and deuterium stay within the statistical uncertainties in both representations.

Since generally sputter yields and sticking coefficients are calculated using the binary collision approximation (BCA) [19], a comparison between the results from the molecular dynamics calculations and the results from SDTrim.SP is important to see which model is applicable in which conditions. Figure 4 shows the net sticking coefficient of several projectile species as a function of the kinetic energy of the projectile. In addition to the results form the molecular dynamics calculations, the results from an SDTrim.SP run with the same sample composition $\left(\mathrm{C}_{0.6} \mathrm{H}_{0.4}\right)$ are plotted. The BCA simulations were performed one atom at a time with the ratio of carbon and hydrogen atoms adjusted to the number of atoms in the projectile molecule. The energies of the 


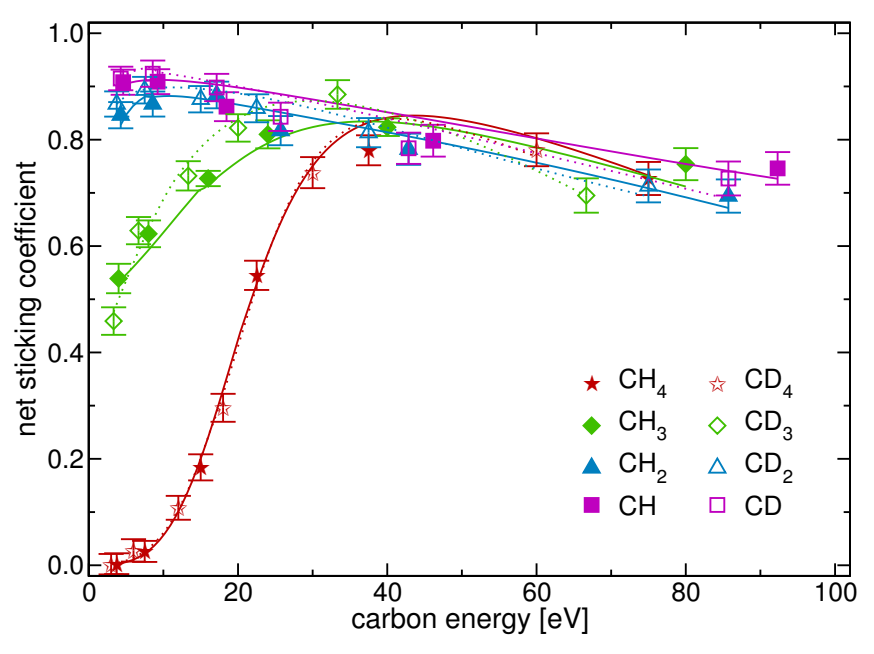

FIG. 3: Net sticking coefficients for $\mathrm{CH}_{x}$ and $\mathrm{CD}_{x}$ projectiles (same data as figure 2) plotted against the energy of the carbon atom in the projectile. The lines represent the interpolation functions discussed in chapter VI with the same parameters as in figure 2 .

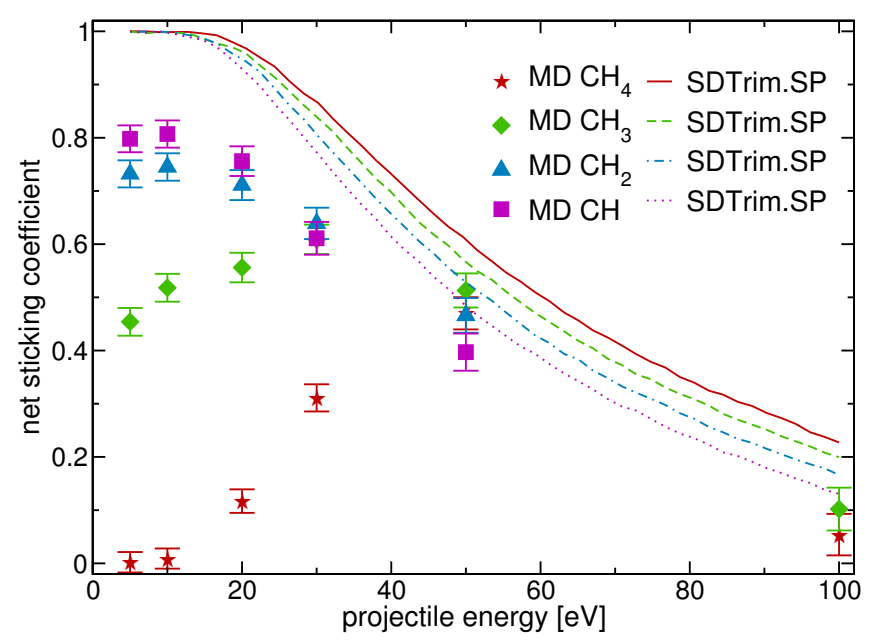

FIG. 4: Net sticking coefficients of various $\mathrm{CH}_{x}$ projectiles, incident at $60^{\circ}$ to the surface normal. Symbols are results form molecular dynamics calculations, solid lines were calculated by SDTrim.SP

atoms were chosen so that the velocity of all impinging atoms matches that of the corresponding molecule. The value used for the surface binding energy $(2.8 \mathrm{eV})$ was chosen according to Jacob et al. [20]

At energies above $50 \mathrm{eV}$, the results from $\mathrm{BCA}$ and $\mathrm{MD}$ are similar with the BCA yielding a higher net sticking coefficient over the whole range. At the low-energy end, the two simulation methods show a completely different behaviour. While the results from the SDTrim.SP calculations converge and all yield a net sticking coefficient of one at the lowest studied energy, the molecular dynamics results approach different, much lower values for differ-

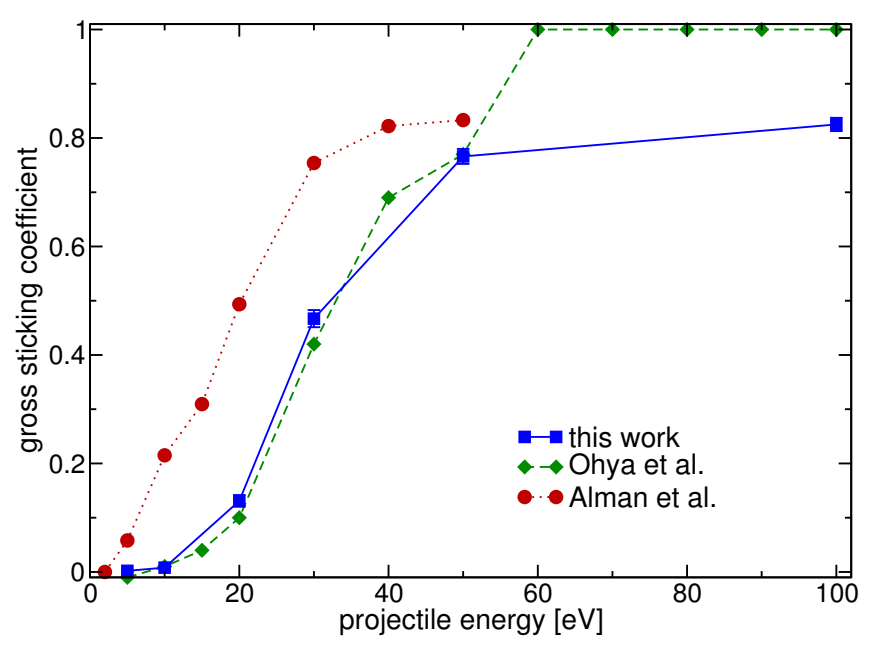

FIG. 5: Comparison of the simulations from this work (squares) with the studies from Alman et al.(circles) and Ohya et al.(diamonds). All data refer to $\mathrm{CH}_{4}$ impinging at an angle of incidence of $45^{\circ}$.

ent projectiles with the lowest $\left(\mathrm{CH}_{4}\right)$ reaching zero at $5 \mathrm{eV}$. This behaviour of the BCA simulations also holds for other angles of incidence. For energies above $50 \mathrm{eV}$, BCA exhibits in general a similar trend as MD but yields always higher net sticking. Since it is well known that $\mathrm{CH}_{4}$ as a stable gas does not stick to walls at ambient temperatures the sticking coefficient of $\mathrm{CH}_{4}$ has to go to 0 at very low energies. Furthermore, the net sticking coefficient of thermal $\mathrm{CH}_{3}$ radicals is about $10^{-4}[21-23]$ so its value should approach values close to 0 at very low energy. Since these two facts strongly support the MD results we assume that MD produces a better description of the behaviour towards low energy than BCA. It is therefore advisable to constrain the use of $\mathrm{BCA}$ results to projectiles energies above $50 \mathrm{eV}$.

Figure 5 shows a comparison between the results from the MD Simulations from this work and the studies by Alman et al. [5] and Ohya et al. [6]. The quantity shown is the gross sticking coefficient, because the net sticking coefficient cannot be reconstructed from the literature data. The results from this work match the data from Ohya et al. [6] quite well up to an energy of $50 \mathrm{eV}$, despite the fact that a different interaction potential was used. Above that energy, all gross sticking coefficients in that work are one. This may result from the fact that above $50 \mathrm{eV}$ most projectiles are emitted as part of hydrocarbon chains with more than one carbon atom (see figure 7 below) that were not considered by Ohya et al. [6].

Alman et al. [5] show a similar saturation value for the gross sticking coefficient as the results from this work, but their value is reached at much lower particle energy. The whole dataset seems to be shifted towards lower energies compared to the studies from Ohya et al. [6] and this work. Since the primary energy of the projectiles is very well defined in $\mathrm{MD}$, it is unclear what causes the 


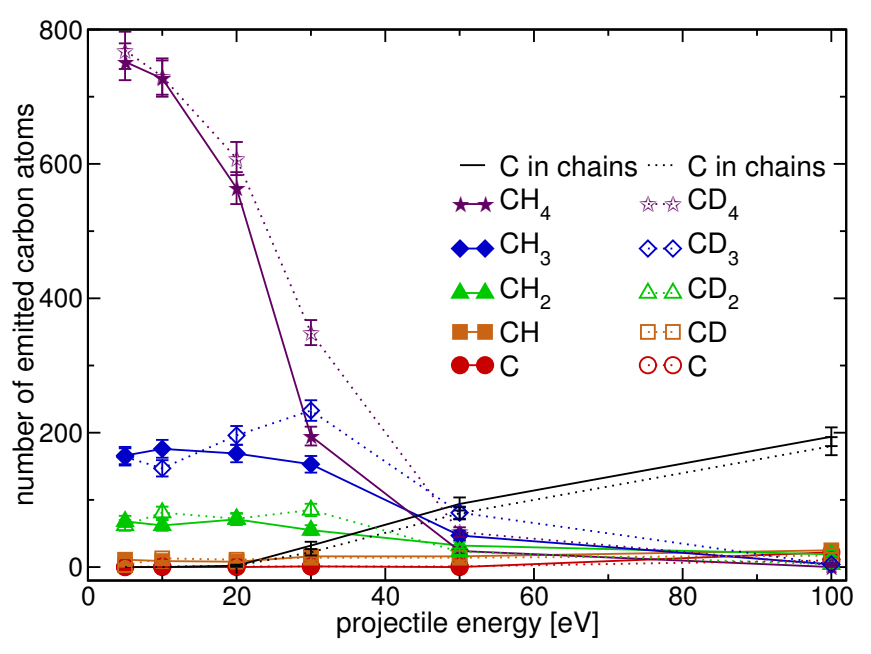

FIG. 6: Abundance of emitted particle species for perpendicular irradiation with $\mathrm{CH}_{4}\left(\mathrm{CD}_{4}\right)$ over a total of 1000 simulation runs

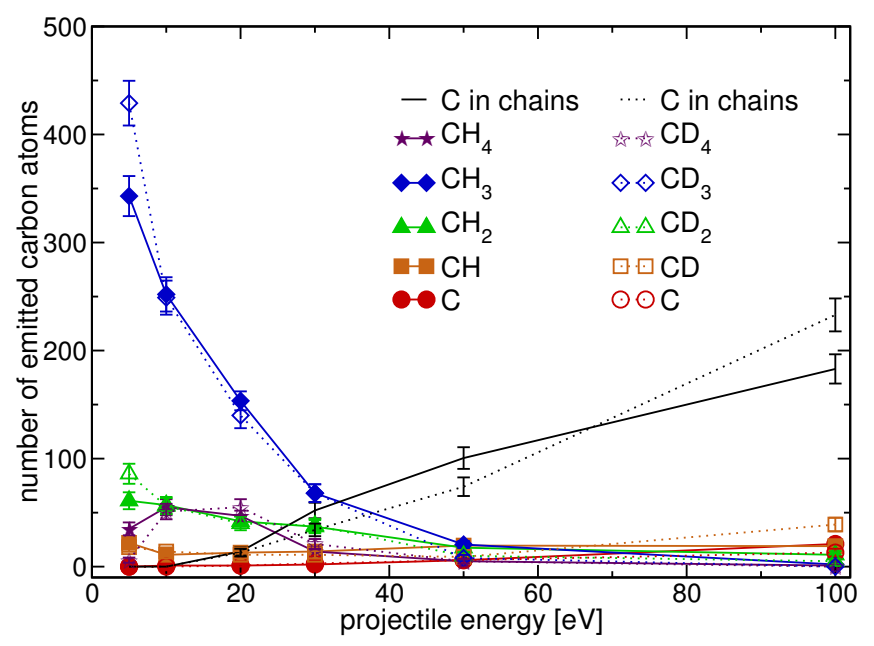

FIG. 7: Abundance of emitted particle species for perpendicular irradiation with $\mathrm{CH}_{3}\left(\mathrm{CD}_{3}\right)$ over a total of 1000 simulation runs

\section{difference.}

In addition to the integral values (sticking coefficient and sputter yield), the molecular dynamics calculations also yield information about the particles that leave the surface. The abundances of different emitted species are plotted in figures 6 and 7 , for $\mathrm{CH}_{4}$ and $\mathrm{CH}_{3}$ as impinging species, respectively. The most frequently emitted species are pure hydrogen species $\left(\mathrm{H}\right.$ and $\left.\mathrm{H}_{2}\right)$ with more than 1000 hydrogen atoms leaving the surface for each set of simulations. Since one is usually interested in carboncontaining species only, the pure hydrogen species were omitted from the plots to improve the readability.

Above the sputtering threshold of about $20 \mathrm{eV}$, many carbon atoms are emitted as part of larger hydrocarbon species. Resulting from the large number of possible
$\mathrm{C}_{x} \mathrm{H}_{y}$ hydrocarbons, the count rate for each of them is very small, although they carry a significant number of carbon atoms. To visualise this fact, only the total number of carbon atoms that left the sample in hydrocarbon chains (molecules with more than one carbon atom) is plotted in figures 6 and 7 .

In both cases, the dominant species up to $50 \mathrm{eV}$ is the projectile species, whereas above that energy, most carbon atoms are emitted as part of hydrocarbon chains. The contribution of individual species depends on the angle of incidence. In average, about $90 \%$ of those chain molecules contain two carbon atoms, this corresponds to about $80 \%$ of the $\mathrm{C}$ atoms in chain molecules. The remaining fraction of chain molecules is, as anticipated, dominated by species carrying $3 \mathrm{C}$ atoms. The largest emitted molecule found in all simulations contained 10 carbon atoms.

For $\mathrm{CH}_{4}$ as the projectile (see figure 6), the number of emitted projectile fragments is almost constant from the lowest studied energies $(5 \mathrm{eV})$ up to $30 \mathrm{eV}$. The second most common fragment is $\mathrm{CH}_{3}$, followed by $\mathrm{CH}_{2}$. Only very few $\mathrm{CH}$ and $\mathrm{C}$ molecules are released at all energies. Above $50 \mathrm{eV}$, the number of emitted projectile fragments drops almost to zero, while a lot of carbon atoms are released in larger hydrocarbon molecules. The emitted species spectrum for $\mathrm{CD}_{4}$ (open symbols) is in general very similar to the $\mathrm{CH}_{4}$ case. Only at about $30 \mathrm{eV}$, the emission pattern of $\mathrm{CH}_{4}$ and $\mathrm{CD}_{4}$ projectiles is different. At this point, the total number of emitted particles is higher for $\mathrm{CD}_{4}$ than for $\mathrm{CH}_{4}$, while the relative amounts of the different species are similar.

For $\mathrm{CH}_{3}$ as projectile (figure 7), the total amount of emitted carbon carrying particles is lower than that for $\mathrm{CH}_{4}$ (figure 6). This is in good agreement with the higher net sticking coefficient presented in figures 2 and 3 . In addition to the projectile fragments also $\mathrm{CH}_{4}$ appears in the emission pattern for projectile energies between $10 \mathrm{eV}$ and $20 \mathrm{eV}$. Outside this energy range, the number of emitted $\mathrm{CH}_{4}$ species is very small. The number of emitted $\mathrm{CH}$ and $\mathrm{C}$ is still negligible at all energies. In the whole energy range the spectrum for $\mathrm{CD}_{3}$ is within the statistical uncertainties identical to that for $\mathrm{CH}_{3}$. The number of carbon atoms that are emitted in larger hydrocarbon molecules is comparable to the data in figure 6 and appears to be almost independent of the projectile species.

\section{ANALYTICAL FIT FUNCTIONS}

The results from the MD simulations fill several gigabytes of compressed data, so for most applications, a smaller and more manageable representation is highly desirable. This is accomplished by fitting an analytical function to the data. That fit function can be used to compute the data very quickly and in addition interpolate the values in between the actual data points. For this process, the gross sticking coefficient and the sputter yield are fitted 
separately and the fit functions are summed up to make a fit function for the net sticking coefficient.

The sputter yields were fitted with the well-established fit formulas by Eckstein [24]:

$$
Y\left(E_{0}\right)=q s_{n}^{K r C}\left(\varepsilon_{L}\right) \frac{\left(\frac{E_{0}}{E_{t h}}-1\right)^{\mu}}{\lambda / w\left(\varepsilon_{L}\right)+\left(\frac{E_{0}}{E_{t h}}-1\right)^{\mu}}
$$

with

$$
\begin{aligned}
s_{n}^{K r C}\left(\varepsilon_{L}\right) & =\frac{1}{2} \frac{\ln \left(1+1.2288 \varepsilon_{L}\right)}{w\left(\varepsilon_{L}\right)} \\
w\left(\varepsilon_{L}\right) & =\varepsilon_{L}+0.1728 \sqrt{\varepsilon_{L}}+0.008 \varepsilon_{L}{ }^{0.1504}
\end{aligned}
$$

It assumes a constant projectile and target mass which is not adequate for molecular projectiles and targets. Therefore for the calculation of the reduced energy

$$
\varepsilon_{L}=E_{0} \frac{M_{2}}{M_{1}+M_{2}} \frac{a_{\mathrm{L}}}{Z_{1} Z_{2} e^{2}}=E_{0} / \varepsilon,
$$

it is assumed that both the projectile (mass $M_{2}$ ) and the target (mass $M_{1}$ ) are pure carbon. The symbol $a_{\mathrm{L}}$ is the Lindhard screening length, given by

$$
a_{\mathrm{L}}=\left(\frac{9 \pi^{2}}{128}\right)^{1 / 3} a_{\mathrm{B}}\left(Z_{1}^{2 / 3}+Z_{2}^{2 / 3}\right)^{-1 / 2}
$$

with the Bohr radius $a_{\mathrm{B}}=0.0529177 \mathrm{~nm}$ and the atomic numbers of target and projectile. The formula for the reduced energy is given in gaussian units. To evaluate it in SI units, $e^{2}$ has to be replaced by $1.4399651 \mathrm{eVnm}$. The resulting value of $\varepsilon$ is $5687 \mathrm{eV}$.

Not only the kinetic energy of the projectile, but also its angle of incidence with respect to the surface normal significantly affects the sticking coefficient and sputtering yield. Data concerning the functional dependence on the angle of incidence using comparable MD simulations were already published [12]. The following fit functions are chosen such that they allow a fit of the energy dependence presented in this article and the already published angular dependence [12]. Eckstein's formula for the angular dependence is well suited for the sputter yields [24]:

$$
Y(E, \alpha)=Y_{0}\left(\cos \left(\left(\alpha / \alpha_{0}\right)^{c}\right)\right)^{-f} \exp \left(b\left(1-\left(\cos \left(\left(\alpha / \alpha_{0}\right)^{c}\right)\right)^{-1}\right)\right)
$$

with

$$
\alpha_{0}=2-\frac{2}{\pi} \arccos \sqrt{\left(1+E / E_{\mathrm{sp}}\right)^{-1}}
$$

Notice that $\alpha_{0}$ is defined different from Eckstein's variant to make the formula simpler (both are equivalent).

Since sticking coefficients and sputter yields are usually required for an arbitrary combination of projectile energy and angle of incidence, a function of both parameters is required for the interpolation. Such a combined function can be created by setting the $Y_{0}$ parameter of the angular

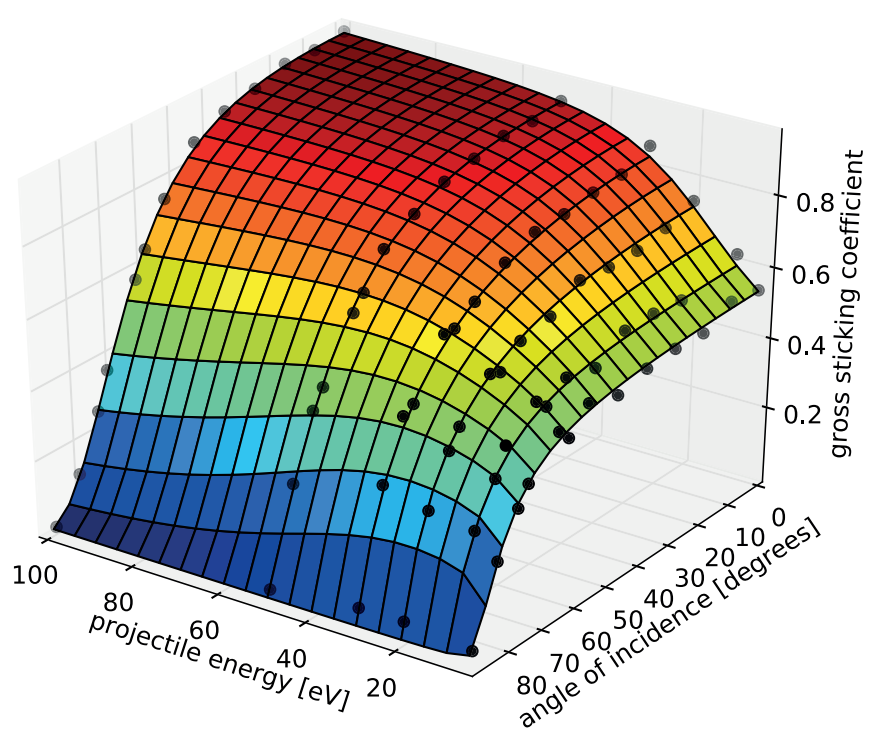

FIG. 8: Three-dimensional representation of the gross sticking coefficient of $\mathrm{CH}_{3}$ as function of both projectile energy and angle of incidence. Solid circles represent the parameter sets where simulations were performed, the surface is the rational bezier spline interpolation to the data

dependence (5) to the result of the energy dependence (1).

In contrast to the sputter yields, the functional dependence of the gross sticking coefficients is not easily separated into energy and angular dependance. The interpolated functional dependency for the gross sticking coefficient of $\mathrm{CH}_{3}$ is shown in figure 8. To represent this data, a third order rational bezier-spline surface [18] was used:

$$
S(E, \theta)=\frac{\sum_{i=0}^{3} \sum_{j=0}^{3} P_{i j} w_{i j} B_{i}(E) B_{j}(\theta)}{\sum_{i=0}^{3} \sum_{j=0}^{3} w_{i j} B_{i}(E) B_{j}(\theta)}
$$

with the basis functions $B_{i}$ derived from the Bernstein polynoms:

$$
B_{i}(x)=B_{i, 3}\left(\frac{x-x_{\min }}{x_{\max }-x_{\min }}\right)
$$

$$
B_{i, 3}(t)=\left(\begin{array}{c}
3 \\
i
\end{array}\right) t^{i}(1-t)^{3-i}
$$

where $x_{\min }$ and $x_{\max }$ are the boundaries of the studied parameter space. For the data given in this paper, this range is $5 \mathrm{eV}$ to $100 \mathrm{eV}$ for the energy and $0^{\circ}$ to $89^{\circ}$ for the angle of incidence. The spline itself does not impose any restrictions on the control points $P_{i j}$. Selecting all weights positive keeps the spline always inside the convex hull of the control points. This simplifies the formulation of the restriction that the gross sticking coefficient cannot be negative and cannot be larger than one by restricting all control points to $[0,1]$. In addition to that, the partial 


\begin{tabular}{c|cccccccc} 
& $q$ & $E_{\text {th }}$ & $\mu$ & $\lambda$ & $f$ & $\alpha_{0}$ & $b$ & $c$ \\
\hline $\mathrm{CH}_{4}$ & 101.273 & 27.485 & 0.617 & 8.923 & 4.166 & 1.577 & 1.969 & 0.823 \\
$\mathrm{CH}_{3}$ & 13.691 & 18.457 & 0.436 & 1.059 & 3.017 & 1.574 & 1.232 & 0.854 \\
$\mathrm{CH}_{2}$ & 35.317 & 9.260 & 0.285 & 2.459 & 2.881 & 1.571 & 1.139 & 0.850 \\
$\mathrm{CH}$ & 1.726 & 4.097 & 0.638 & 0.242 & 5.312 & 1.711 & 2.473 & 0.857 \\
\hline $\mathrm{CD}_{4}$ & 167.029 & 27.310 & 0.562 & 15.703 & 11.864 & 2.122 & 7.984 & 0.649 \\
$\mathrm{CD}_{3}$ & 15.132 & 17.065 & 0.704 & 1.745 & 6.333 & 1.877 & 3.735 & 0.631 \\
$\mathrm{CD}_{2}$ & 1.876 & 0.869 & 1.060 & 4.453 & 7.150 & 2.169 & 4.290 & 0.674 \\
$\mathrm{CD}$ & 26.450 & 9.842 & 0.229 & 1.645 & 9.261 & 2.090 & 5.503 & 0.853
\end{tabular}

TABLE I: Fit parameters for the sputtering formulas (1) and (5)

derivative with respect to the angle of incidence is forced to zero at perpendicular incidence by setting the first two control points and weights in that direction to the same value:

$$
\begin{aligned}
& P_{i 0}=P_{i 1} \\
& w_{i 0}=w_{i 1}
\end{aligned}
$$

This results in 24 fit parameters: 12 control points $P_{0 . \ldots 3,1 \ldots 3}$ and 12 weights $w_{0, \ldots 3,1 \ldots 3}$. The figures 1 to 3 contain the graphs of the fit functions, drawn as solid (protium) and dotted (deuterium) lines. The parameters of these functions were fitted to the data from the MD simulations by minimising $\chi^{2}$, taking into account the effect of the statistical uncertainties of the simulation data. Within these statistical uncertainties, the analytical formulas fit the data quite well and provide a smooth interpolation of the data points. A collection of the fit parameters is compiled in tables I and II.

\section{CONCLUSIONS}

Simulations were performed to determine the sticking coefficient and the emission patterns of $\mathrm{C}_{1}$ hydrocarbons with protium and deuterium on an amorphous hydrocarbon surface. It was found that the differences between the protinated and the deuterated species are very small over the studied range of incident energies ( 5 to $100 \mathrm{eV}$ ). For projectile energies as low as $5 \mathrm{eV}, \mathrm{C}, \mathrm{CH}$ and $\mathrm{CH}_{2}$ show about $90 \%$ net sticking, $\mathrm{CH}_{3}$ has $50 \%$ sticking while the sticking of $\mathrm{CH}_{4}$ is almost zero. At projectile energies above $50 \mathrm{eV}$, most carbon atoms leave the sample surface as part of multi-carbon hydrocarbon molecules. The selection of hydrogen isotope has only very little influence on sticking coefficient and sputtering yield. A comparison with results from $\mathrm{BCA}$ simulations shows that the BCA should not be used at energies below $50 \mathrm{eV}$ for sticking calculations. The data were fitted to analytical functions with the parameters compiled in tables I and II for interpolation and lookup of data using functions of both energy and angle of incidence, thereby greatly facilitating the access to the data for use in transport simulations.
[1] ITER Technical Basis. ITER EDA Documentation Series. International Atomic Energy Agency, Wien, 2002.

[2] W. Jacob. Redeposition of hydrocarbon layers in fusion devices. J. Nucl. Mater., 337-339:839-846, 2005.

[3] A. Kirschner, K. Ohya, D. Borodin, R. Ding, D. Matveev, V. Philipps, and U. Samm. Prediction of long-term tritium retention in the divertor of ITER: influence of modelling assumptions on retention rates. Phys. Scripta, T138:014011 (6pp), 2009.

[4] Y. Mu, A.G. McLean, J.D. Elder, P.C. Stangeby, B.D. Bray, N.H. Brooks, J.W. Davis, M.E. Fenstermacher, M. Groth, C.J. Lasnier, D.L. Rudakov, J.G. Watkins, W.P. West, and C.P.C. Wong. 3D-DIVIMP-HC modeling analysis of methane injection into DIII-D using the DiMES porous plug injector. Journal of Nuclear Materials, 390-391(0):220 - 222, 2009.

[5] D. A. Alman and D. N. Ruzic. Molecular dynamics simulation of hydrocarbon reflection and dissociation coefficients from fusion-relevant carbon surfaces. Phys. Scripta, T111:145-151, 2004.

[6] K. Ohya, Y. Kikuhara, K. Inai, A. Kirschner, D. Borodin, A. Ito, H. Nakamura, and T. Tanabe. Simulation of hydrocarbon reflection from carbon and tungsten surfaces and its impact on codeposition patterns on plasma facing components. Journal of Nuclear Materials, 390-391:72 75, 2009.

[7] D. W. Brenner. Empirical potential for hydrocarbons for use in simulating the chemical vapor deposition of diamond films. Phys. Rev. B, 42:9458, 1990.

[8] N. Juslin, P. Erhart, P. Träskelin, J. Nord, K. O. E. Henriksson, K. Nordlund, E. Salonen, and K. Albe. Analytical interatomic potential for modeling nonequilibrium processes in the $\mathrm{W}-\mathrm{C}-\mathrm{H}$ system. Journal of Applied Physics, 98(12):123520, 2005.

[9] T. Schwarz-Selinger, A. von Keudell, and W. Jacob. Plasma chemical vapor deposition of hydrocarbon films: The influence of hydrocarbon source gas on the film properties. J. Appl. Phys., 86:3988, 1999.

[10] J. Robertson. Diamond-like amorphous carbon. Mat. Sci. Eng. Reports, 37:129, 2002.

[11] P. N. Maya, U. von Toussaint, and C. Hopf. Synergistic erosion process of hydrocarbon films: A molecular dynamics study. New J. Phys., 10:023002 (15pp), 2008.

[12] K. Tichmann, U. von Toussaint, and W. Jacob. Fit formulas for the angular dependence of the sticking coefficient of energetic hydrocarbon molecules. Journal of Nuclear Materials, 415 (1, Supplement):S196, 2010.

[13] P. S. Krstić, C. O. Reinhold, and S. J. Stuart. Chemical sputtering by impact of excited molecules. Europhys. Lett., 77:33002 p1-p6, 2007.

[14] A. Sharma, R. Schneider, U. von Toussaint, and K. Nordlund. Hydrocarbon radicals interaction with amorphous carbon surfaces. J. Nucl. Mater., 363-365:1283-1288, 2007. 


\begin{tabular}{|c|c|c|c|c|c|c|}
\hline & \multicolumn{3}{|c|}{$P$} & \multicolumn{3}{|c|}{$w$} \\
\hline \multirow{4}{*}{$\mathrm{CH}$} & 0.91 & 0.89 & 0.24 & 0.10 & 1.29 & 0.37 \\
\hline & 0.93 & 1.00 & 0.56 & 1.69 & 1.67 & 0.77 \\
\hline & 0.00 & 0.92 & 0.00 & 0.14 & 7.59 & 2.11 \\
\hline & 0.97 & 1.00 & $0.02)$ & 37.25 & 10.37 & $22.41)$ \\
\hline \multirow{4}{*}{$\mathrm{CH}_{2}$} & 0.84 & 0.03 & 0.09 & $(2.14$ & 0.15 & 0.02 \\
\hline & 1.00 & 0.88 & 0.08 & 3.92 & 11.34 & 2.53 \\
\hline & 0.00 & 0.72 & 0.00 & 1.17 & 10.72 & 0.00 \\
\hline & 0.96 & 0.89 & $0.00 /$ & 90.28 & 17.94 & $42.01)$ \\
\hline \multirow{4}{*}{$\mathrm{CH}_{3}$} & 0.54 & 0.13 & 0.02 & 6.23 & 0.38 & 0.21 \\
\hline & 1.00 & 0.63 & 0.00 & 4.17 & 9.21 & 2.85 \\
\hline & 0.88 & 1.00 & 0.77 & 20.40 & 5.73 & 0.00 \\
\hline & 0.96 & 0.37 & $0.00 /$ & 114.70 & 12.32 & 35.88 \\
\hline \multirow{4}{*}{$\mathrm{CH}_{4}$} & 0.00 & 0.00 & 0.00 & 4.33 & 3.31 & 3.02 \\
\hline & 0.09 & 0.00 & 0.00 & 0.00 & 0.00 & 0.00 \\
\hline & 0.70 & 1.00 & 0.00 & 5.96 & 3.48 & 0.00 \\
\hline & 0.95 & 0.08 & $0.00 /$ & 110.93 & 39.72 & $36.08)$ \\
\hline \multirow{4}{*}{$C D$} & 0.91 & 0.72 & 0.58 & $(0.13$ & 0.03 & 0.04 \\
\hline & 0.12 & 0.95 & 0.63 & 0.00 & 16.38 & 8.58 \\
\hline & 0.94 & 1.00 & 0.00 & 51.29 & 1.50 & 16.87 \\
\hline & 0.96 & 1.00 & 0.14 & 45.18 & 3.03 & 25.79 \\
\hline \multirow{4}{*}{$\mathrm{CD}_{2}$} & 0.87 & 0.84 & 0.00 & 0.11 & 1.49 & 0.46 \\
\hline & 0.89 & 0.92 & 0.22 & 8.89 & 5.81 & 0.85 \\
\hline & 0.94 & 0.45 & 0.00 & 45.60 & 16.51 & 0.00 \\
\hline & 0.96 & 1.00 & 0.00 & 95.64 & 36.58 & 68.03 \\
\hline \multirow{4}{*}{$\mathrm{CD}_{3}$} & 0.47 & 0.24 & 0.01 & 8.65 & 4.84 & 0.26 \\
\hline & 1.00 & 0.06 & 0.45 & 14.83 & 0.55 & 12.26 \\
\hline & 1.00 & 0.88 & 0.26 & 15.94 & 0.01 & 0.01 \\
\hline & 0.95 & 0.33 & $0.00)$ & 61.52 & 3.81 & $19.85)$ \\
\hline \multirow{4}{*}{$\mathrm{CD}_{4}$} & 0.00 & 0.00 & 0.00 & 10.11 & 20.29 & 20.14 \\
\hline & 0.28 & 0.00 & 0.00 & 3.28 & 0.00 & 0.00 \\
\hline & 0.00 & 0.68 & 0.00 & 0.00 & 19.35 & 0.00 \\
\hline & 0.96 & 0.58 & $0.00)$ & 120.75 & 9.86 & $52.29)$ \\
\hline
\end{tabular}

TABLE II: Fit parameters for the spline fit of the sticking coefficients.
[15] Keith Beardmore and Roger Smith. Empirical potentials for $\mathrm{C}-\mathrm{Si}-\mathrm{H}$ systems with application to $\mathrm{C}_{60}$ interactions with Si crystal surfaces. Philosophical Magazine A, 74(6):1439-1466, 1996.

[16] K. Nordlund, J. Keinonen, and T. Mattila. Formation of ion irradiation induced small-scale defects on graphite surfaces. Phys. Rev. Lett., 77(4):699-702, 1996.

[17] W. Eckstein and R. Preuss. New fit formulae for the sputtering yield. J. Nucl. Mater., 320:209-213, 2003.

[18] L.A. Piegl and W. Tiller. The NURBS book. Monographs in visual communication. Springer, 1997.

[19] W. Eckstein. Computer simulation of ion-solid interactions. Springer Series in Materials Science. Springer Verlag, Berlin and Heidelberg, 1 edition, 1991.

[20] W. Jacob, C. Hopf, and M. Schlüter. Chemical sputtering of carbon materials due to combined bombardment by ions and atomic hydrogen. Phys. Scripta, T124:32-36, 2006.

[21] A. von Keudell, T. Schwarz-Selinger, M. Meier, and W. Jacob. Direct identification of the synergism between methyl radicals and atomic hydrogen during growth of amorphous hydrogenated carbon films. Appl. Phys. Lett., $76: 676,2000$.

[22] A. von Keudell, T. Schwarz-Selinger, M. Meier, and W. Jacob. Erratum: Direct identification of the synergism between methyl radicals and atomic hydrogen during growth of amorphous hydrogenated carbon films. Appl. Phys. Lett., 77:459, 2000.

[23] A. von Keudell, T. Schwarz-Selinger, and W. Jacob. Simultaneous interaction of methyl radicals and atomic hydrogen with amorphous hydrogenated carbon films. $J$. Appl. Phys., 89:2979, 2001.

[24] W. Eckstein and H. M. Urbassek. Computer simulation of the sputtering process. In R. Behrisch and W. Eckstein, editors, Sputtering by Particle Bombardment IV, volume 110 of Topics in Applied Physics, chapter Computer Simulation of the Sputtering Process, pages 21-31. Springer Verlag, Berlin, 2007. 